\title{
Buoyancy-Driven Instabilities in Single-Bubble Sonoluminescence
}

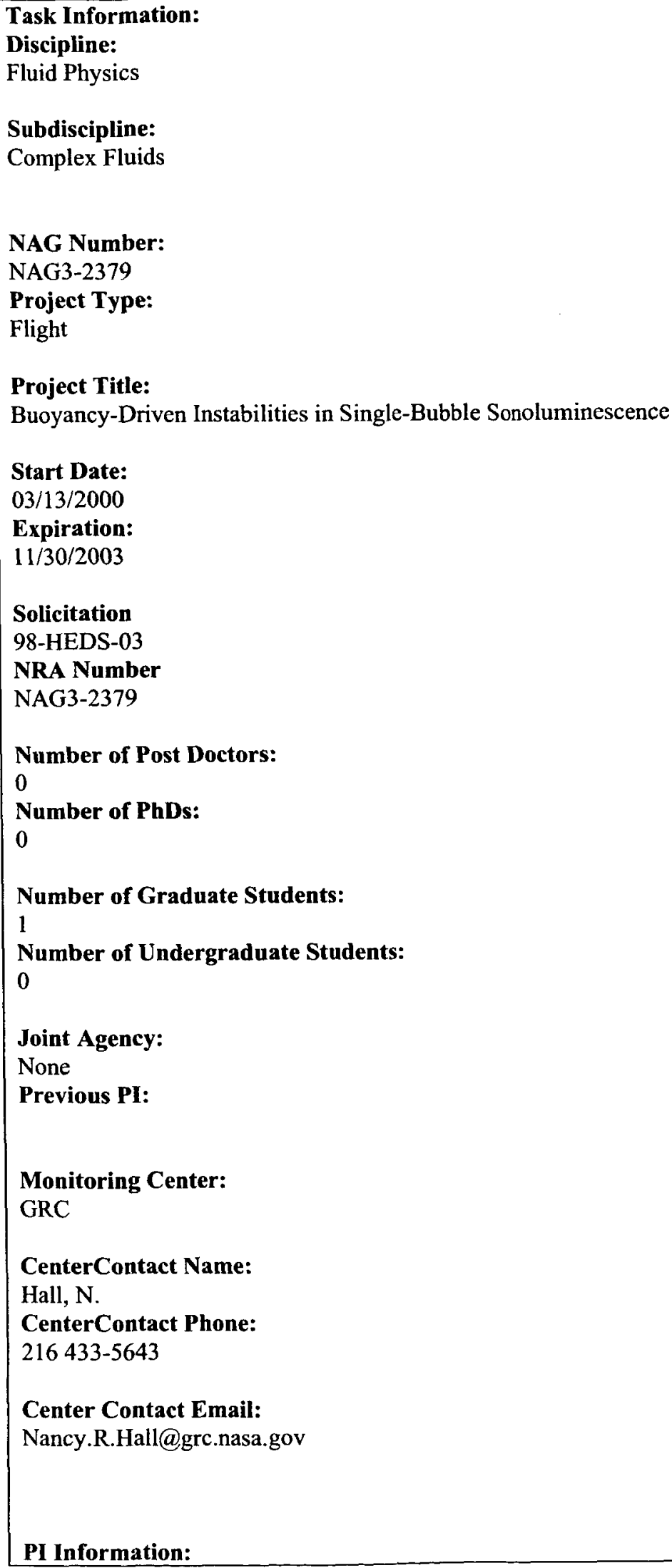




\title{
Buoyancy-Driven Instabilities in Single-Bubble Sonoluminescence
}

\author{
Name: \\ Matula, Thomas J. \\ Phone: \\ 206-685-7654 ext \\ Email: \\ matula@apl.washington.edu \\ Fax: \\ 206-543-6785 \\ Institution Type: \\ University \\ Institution: \\ University of Washington \\ Address1: \\ Applied Physics Lab \\ Address2: \\ 1013 NE 40th St. \\ City: \\ Seattle \\ State: \\ WA \\ Zipcode: \\ 98105-6698

\section{Congressional District:} \\ 7 \\ Comments: \\ Acronym: \\ SL \\ sonoluminescence
}

\section{Task Abstract/Description:}

The principal objectives of this study are to determine how gravity affects the emission of light from single-bubble sonoluminescence (SBSL), and whether or not the bubble extinction is directly related to gravity. Our experimental task involves designing glass or quartz spherical levitation cells that generate very stable SL bubbles. The cells must have minimized vibration, and some temperature control. The experimental system will reside in a light-tight enclosure. Aside from acceleration, the frequency, pressure amplitude, and light intensity must be measured. A computer program will be constructed to perform all aspects of the experiment. 


\section{Buoyancy-Driven Instabilities in Single-Bubble Sonoluminescence}

\section{Task Objective:}

The object of this research program is to quantify buoyancy-induced instabilities that may play a dominant role in the mechanism for sonoluminescence extinction. Such instabilities should be manifest in the longterm stability of the sonoluminescence bubble, and in the maximum forcing amplitude that can sustain stable sonoluminescence. Thus, light-emission data will be recorded under two conditions. (1) During constant forcing conditions, whereby the long-term stability of the light emission will be recorded, and (2) during a gradual approach to the extinction threshold. Furthermore, the bubble size (diameter) must also be measured, at its equilibrium (or natural) diameter, and again at its maximum diameter. These measurements will then be used in simulations of bubble dynamics to test current models for bubble extinction.

\section{Task Significance:}

The significance of this research goes far beyond the physics research laboratory. Determining how instabilities affect sonoluminescence will lead to understanding the mechanism for sonoluminescence. Understanding the role of instabilities in sonoluminescence, possible to the observance of hard x-ray emission, or even fusion, albeit over small temporal and spatial scales. The potential uses include a tabletop experimental device that can be used to test against computer simulations for implosions, such at those used in nuclear research centers. Furthermore, these studies should help in understanding the relatively new field of sonochemistry, which relies on cavitation bubbles for initiating or facilitating chemical reactions. Other uses not yet dreamed of, because of the current limitations, may become apparent if cavitation bubbles can concentrate more energy. At the very least, there exist the potential of exotic physics that can occur under the extreme conditions that sonoluminescence bubble experience, provided the energy concentration can be increased.

Impact on America:

\section{Industry Affiliates:}

\section{Invovative Technologies Developed:}

With, Burle Inc., we developed a high-speed spectral PMT that covers the range of 250-600 nm.

\section{Research Audience:}

\section{Graduate Student Employment:}

5. No. times Research presented on Magazine Cover: 


\section{Buoyancy-Driven Instabilities in Single-Bubble Sonoluminescence}

\section{Research impact on American/Earth Benefits:}

Task Progress:

Modeling effort: The modeling effort has been very successful. We have implemented a model to describe the translation of a bubble trapped in a standing acoustic wave field. The results of that effort led to a manuscript being submitted for publication. After considering the comments of the reviewers', the manuscript was revised and re-submitted. It was accepted, and finally published in a prestigeous peerreviewed journal (see bibliography for details).

Experimental effort: The experimental effort has been steady. With considerable help from the engineering staff devoted to this project, we have designed and constructed levitation chambers that are repeatable and consistent. Passing this major milestone has been very important. The other important milestone has been the design and construction of a spectral photomultiplier tube (PMT) with an ability to measure the calibrated intensity of a sonoluminescing bubble.

Reviews: During the past year we underwent the Requirements Definition Review (RDR) and Preliminary Design Review (PDR). The Science Requirement Document (SRD) was revised after the RDR, and is now being implemented by the engineering team.

\section{COI Information:}

\section{COI Name \\ COI Institution}

Crum, Lawrence A

University of Washington

Bibiography Information:

\section{Bibliography Type \\ Bibliography Description}

Proceeding Entries

T. J. Matula. Buoyancy-driven instabilities in single-bubble sonoluminescence. in Conference and Exhibit on International Space Station Utilization. 2001. Cape Canaveral: American Institute of Aeronautics and Astronautics.

Articles in Peer-reviewed Journals

1. T. J. Matula, Single-bubble sonoluminescence in microgravity. Ultrasonics, 2000. 38: p. 559-565. 


\section{Buoyancy-Driven Instabilities in Single-Bubble Sonoluminescence}

2. T. J. Matula, et al., The effects of buoyancy on sonoluminescing bubbles. ARLO, 2000. 1(1): p. 13-18.

3. T. J. Matula, Bubble levitation and translation under single-bubble sonoluminescence conditions. Journal of the Acoustical Society of America, 2003. 114(2): p. 775-781.

4. 1. J. A. Reed, et al., The effects of microgravity on nanoparticle size distributions generated by the ultrasonic reduction of an aqueous gold-chloride solution. Ultrasonics Sonochemistry, 2003. 10: p. 285-289. 